\title{
C-E TYPE TOEPLITZ OPERATORS ON $L_{a}^{2}(\mathbb{D})$
}

\author{
M. R. JABBARZADEH AND M. MORADI
}

Abstract. In this paper, we initiate the study of a new class of conditional type operators, which we call C-E type Toeplitz operators. Sufficient conditions for boundedness and compactness of C-E type Toeplitz operators on the Bergman space $L_{a}^{2}(\mathbb{D})$ will be presented. Also, some differences between C-E type Toeplitz operators and Toeplitz operators will be illustrated by examples.

Mathematics subject classification (2010): Primary 47B20, Secondary 47B38.

Keywords and phrases: Bergman space, Toeplitz operator, conditional expectation.

\section{REFERENCES}

[1] A. B. Aleksandrov, Measurable partitions of the circle induced by inner functions, J. Soviet Math. 42 (1988), 1610-1613.

[2] K. R. M. ATtElE, Multipliers of the range of composition operators, Tokyo J. Math. 15 (1992), $185-198$.

[3] J. A. BALL, Hardy space expectation operators and reducing subspaces, Proc. Amer. Math. Soc. 47 (1975), 351-357.

[4] B. J. Carswell and M. L. Stessin, Conditional expectation and the Bergman projection, J. Math. Anal. Appl. 341 (2008), 270-275.

[5] P. G. Dodds, C. B. Huijsmans and B. De Pagter, Characterizations of conditional expectationtype operators, Pacific J. Math. 141 (1990), 55-77.

[6] J. J. GROBLER AND B. DE PAGTER, Operators representable as multiplication-conditional expectation operators, J. Operator Theory 48 (2002), 15-40.

[7] H. Hedenmalm, B. Korenblum and K. Zhu, Theory of Bergman spaces, Springer-Verlag, New York, 2000.

[8] M. R. JabBarzadeh AND M. Hasanloo, Conditional expectation operators on the Bergman spaces, J. Math. Anal. Appl. 385 (2012), 322-325.

[9] I. Louhichi, E. Strouse And L. Zakariasy, Products of Toeplitz operators on the Bergman space, Integr. Equ. Oper. Theory 54 (2006), 525-539.

[10] J. N. MCDonald, Adjoints of a class of composition operators, Proc. Amer. Math. Soc. 131 (2003), 601-606.

[11] K. StRoethofF, The Berezin transform and operators on space of analytic functions, Polish academy of science, Warszawa, 1997.

[12] M. M. RaO, Conditional measures and applications, Marcel Dekker, New York, 1993.

[13] A. C. ZAAnEN, Integration, 2nd ed., North-Holland, Amsterdam, 1967.

[14] K. ZHU, Operator theory in function spaces, Marcel Dekker, New York 1990. 